RAEIC, Revista de la Asociación Española de Investigación de la Comunicación

\title{
La gestión de los medios sociales en los destinos turísticos según sus directores de comunicación
}

Social media management in tourist destinations according to their communication directors

Pastor-Marín, Francisco-Manuel

Universidad de Málaga (UMA) franciscopastor@gmail.com

Paniagua-Rojano, Francisco-Javier Universidad de Málaga (UMA) fjpaniagua@uma.es

Cabrera-González, Mángeles Universidad de Málaga (UMA) mac@uma.es

Forma de citar este artículo:

Pastor-Marín, F. M., Paniagua-Rojano, F. J., y Cabrera-González, M. A. (2020). “La gestión de los medios sociales en los destinos turísticos según sus directores de comunicación", RAEIC, Revista de la Asociación Española de Investigación de la Comunicación, vol. 7, núm. 13, 213-230 https://doi.org/10.24137/raeic.7.13.10 


\section{Resumen:}

El objeto de este estudio es analizar cómo los destinos turísticos gestionan los medios sociales y cuál es la labor de sus directores de comunicación para saber qué valoración hacen de su presencia en la red. Se trata, en definitiva, de estudiar la planificación de la comunicación digital de los destinos turísticos según el punto de vista de sus responsables. Para ello, esta investigación, basada en el estudio de diferentes destinos urbanos de todo el mundo, se apoya en el método cualitativo, al realizar entrevistas a los responsables de comunicación de estos destinos con el fin de identificar su papel en la estructura del departamento, sus funciones y necesidades. Los resultados muestran un avance significativo en la formación de los directores de comunicación y se comprueba que los destinos turísticos empiezan a usar los medios sociales como herramienta de diálogo e interacción con sus públicos, aunque en general la utilización de las redes sigue siendo más intuitiva que estratégica y se concluye que los destinos tienen ahora en los medios sociales su principal herramienta comunicativa.

Palabras clave: comunicación, medios sociales, destinos turísticos, dircom, públicos.

\section{Abstract:}

The purpose of this study is to analyze how tourist destinations manage social media and what is the work of their communication directors to know what assessment they make of their presence on the network. It is, in short, to study the planning of digital communication of tourist destinations according to the point of view of those responsible. For this, this research, based on the study of different urban destinations around the world, relies on the qualitative method, by conducting interviews with those responsible for communicating these destinations in order to identify their role in the structure of the department, functions and needs. The results show a significant advance in the training of communication directors and it is proven that tourist destinations begin to use social media as a tool for dialogue and interaction with their audiences, although in general the use of networks remains more intuitive than strategic 
and it is concluded that destinations now have their main communicative tool in social media.

Keywords: communication, social media, tourist destinations, communication directors, audience.

\section{INTRODUCCIÓN}

El turismo ha experimentado un crecimiento constante en los últimos años y se ha convertido en una industria líder en todo el mundo (UNWTO, 2018). Esta evolución ha estado acompañada por el auge de las Tecnologías de la Información y la Comunicación (TIC) en general e Internet en particular (Buhalis y Law, 2008), aunque ha sido la aparición y posterior desarrollo de los medios sociales, considerados como "el modus operandi del siglo XXI" (Lange-Faria y Elliot, 2012), los que han transformado la comunicación turística (Xiang y Gretzel, 2010; De San Eugenio 2011; Míguez, MarinéRoig y Huertas, 2014; Munar y Jacobesen, 2014; Wichels, 2014; Paniagua y Huertas, 2018).

Esto ha sido así debido fundamentalmente a la gran capacidad que tienen los medios sociales de interacción y de creación de diálogo con sus públicos (Lim, Chung y A Weaber, 2012; Míguez et al., 2014; Altamirano y Túñez López, 2016). De hecho, los medios sociales juegan un gran papel como fuente de información para el turista (Xiang y Gretzel, 2010; Miguéns, Baggio y Costa, 2008) y han contribuido a cambiar el paradigma de la comunicación, situando al turista en el centro, de forma que el turismo tradicional, organizado y uniformador, ha dado paso al turismo experiencial (De San Eugenio, 2011). Los destinos se benefician de estos medios en la fase previa al viaje, cuando buscan información sobre el lugar que van a visitar; durante el viaje mismo, cuando publican y comparten información; y al volver a sus hogares, cuando comparten las experiencias vividas (Mangold y Faulds, 2009; Mendes Thomaz, Augusto Biz y Gândara, 2013). De hecho, ya se habla de la aparición de un nuevo viajero, que ha 
Pastor-Marín, Francisco-Manuel, Paniagua-Rojano, Francisco-Javier, y Cabrera-González, María-Ángeles

decidido adquirir sus productos turísticos de forma directa y sin intermediarios (Buhalis y Law, 2008).

\section{REVISIÓN DE LITERATURA}

La elección de un destino supone un elemento de riesgo debido a la intangibilidad del propio sector. Este riesgo puede ser minimizado por las opiniones de otros turistas (Mill y Morrison, 2012; Mendes Thomas et al., 2013), y sus valoraciones serán tenidas en cuenta más que las de las Oficinas de Marketing de Destino (OMD) (Xiang y Gretzel, 2010; Domínguez y Araújo, 2012).

Además, los destinos turísticos están más necesitados que nunca de comunicar. La marca se ve ahora como un elemento propio de comunicación (Fernández-Cavia, 2011). Los territorios con una marca potente tienen más fácil atraer a los turistas, pero también a nuevos residentes o futuras inversiones (Morgan, Pritchard y Pride, 2011). Aunque un territorio no es un producto, la mayor parte de los estudios (Fernández-Cavia y Huertas, 2009) consideran que el branding también puede aplicarse a los territorios. De hecho, la marca-territorio es ahora lo que más identifica a un destino turístico (De San Eugenio, 2011).

Los medios sociales son unas herramientas con un gran potencial para las relaciones públicas, que entienden la comunicación como un elemento bidireccional entre la organización y sus públicos (Fernández-Cavia y Huertas, 2014). Su uso permite no sólo saber lo que piensan los viajeros sino también las Organizaciones de Marketing de los Destinos (OMD) puedan entablar diálogo con ellos y abrir la puerta a relaciones futuras (Wigley y Lewis, 2012; Fernández-Cavia y Huertas, 2014; Mariné-Roig y Huertas, 2016). Por ello, los medios sociales ofrecen múltiples oportunidades a las instituciones turísticas $(X u, 2010)$ y contribuyen al establecimiento de la marca del destino (Domínguez y Araújo, 2012).

Lógicamente, este diálogo se crea con una buena gestión de los medios sociales, permitiendo los comentarios y contestándolos. Si sólo se usan las herramientas de forma unidireccional es imposible que se dé el diálogo (Míguez et al., 2014). No en vano 
aunque los propios medios sociales, por su tecnología, tienen en sí mismos esa capacidad de interacción (Lovejoy y Saxton, 2012), para que ésta sea efectiva es imprescindible que los gestores de los medios sociales las usen de forma correcta (Zhou y Wang, 2013). Algunos autores (Míguez et al., 2014; Martínez-Sala y Campillo-Alhama, 2018) señalan, sin embargo, que las OMD no están aprovechando todo el potencial que los medios sociales tienen como herramienta para conseguir diálogo con sus públicos. Y ello pese a que el establecimiento de relaciones entre las OMD y los diferentes usuarios es quizá uno de los aspectos más trascendentes de los medios sociales (Altamirano y Túñez-López, 2016).

De esta forma, si bien es cierto que los medios sociales han ofrecido nuevas oportunidades, también han supuesto un reto importante para las instituciones turísticas, así como para los directores de comunicación y los propios gestores de los medios, que en poco tiempo se han tenido que adaptar a una realidad nueva y cambiante. Por lo general, las OMD se están adaptando a esta transformación con gran rapidez y profesionalidad, aunque debido a la celeridad con la que se producen estos cambios, en muchas ocasiones los destinos turísticos están usando los medios sociales sin saber bien en cuáles estar presentes o cuáles son los efectos de sus estrategias, de forma más intuitiva que estratégica (Hvass y Munar, 2012; Hays, Page y Buhalis, 2013).

Todavía hoy se usan estos medios para informar, sin dialogar (Fernández Cavia y Huertas, 2009), por miedo a las críticas o por la falta de formación (Huertas, 2014). Aun así, los medios sociales son el canal más importante que encuentran los dircom para dirigirse a sus públicos y la relevancia queda reflejada en la importancia que les otorgan los propios directores de comunicación. El 90,4\% de los dircom considera que los medios sociales son ya el canal más importante (Zerfass, Moreno, Tech, Vercic y Verhoeven, 2017), por delante de websites, periódicos online, eventos, canales de radio y televisión o periódicos tradicionales. De esta forma, la percepción de la importancia de los medios sociales ha pasado del 10\% en 2007 a más del 90\% una década más tarde.

De hecho, los destinos apuestan cada vez más por este tipo de comunicación, lo que ha conllevado incluso cambios en la estructura de la mayoría de los departamentos de 
comunicación y marketing de los destinos (Wilches, 2014). No en vano, el uso de los medios sociales obliga a los responsables de comunicación de los destinos a un continuo esfuerzo de actualización (Domínguez y Araújo, 2012).

A pesar de la amplia adopción de los medios sociales por parte tanto de los consumidores como de los proveedores turísticos en los últimos años, la práctica exitosa de manipular y administrar estos medios sigue siendo en gran parte desconocida para los profesionales y los académicos (Leung, Law, van Hoof y Buhalis, 2013). Aunque el estudio de los medios sociales en el contexto turístico es un área creciente de investigación (Lange-Faria y Elliot, 2012), hay pocos estudios sobre la aplicación de los medios sociales por parte de las organizaciones turísticas (Xiang y Gretzel, 2010; Hays et al., 2013; Huertas, Setó y Míguez, 2015) y los que existen se han centrado sobre todo en el aspecto cuantitativo, poniendo de manifiesto que los destinos turísticos cada vez usan más los medios sociales y de ahí la importancia de una buena coordinación; una coordinación que debe ir acompañada de la colaboración de todos los responsables de la creación de contenidos, y que estos aporten valor durante todo el proceso (Paniagua y Huertas, 2018).

De esta forma, se hace imprescindible saber cuáles son las estrategias que usan los responsables de comunicación de los destinos turísticos para comunicar sus marcas a través de los medios sociales a fin de que esta comunicación sea lo más útil posible para sus viajeros. Sólo conociendo qué se está haciendo con respecto a la gestión de los medios sociales podrá establecerse una perspectiva más amplia que apunte de forma real por la interacción con los turistas y viajeros.

\section{METODOLOGÍA}

El objetivo de este trabajo es conocer cómo los grandes destinos turísticos internacionales gestionan su comunicación y especialmente el uso de los medios sociales con el fin de que esta comunicación sea útil. Además, pretende analizar el trabajo de gestión de los responsables de comunicación, cuáles son sus funciones, qué tareas concretas llevan a cabo y qué valoración hacen de su presencia en la red para finalmente ofrecer una propuesta de actuación. En definitiva, se trata de estudiar la 
planificación de la comunicación digital de los destinos turísticos según el punto de vista de sus responsables. Para conseguirlo, se ha optado por el uso de una metodología cualitativa que permita dar respuestas en profundidad a las cuestiones planteadas: la entrevista a los directores de comunicación de destinos objeto de nuestro estudio.

La selección de la muestra coincide con los 15 destinos del estudio 'City Tourism Performance Research' (UNWTO, 2017), que recoge los casos de éxito y buenas prácticas realizados en algunos de los principales destinos turísticos urbanos del mundo, a los que se suma Málaga por ser destino que más crece en España. Así, conforman la muestra final Málaga, Amberes, Berlín, Bogotá, Buenos Aires, Copenhague, Ciudad del Cabo, Hangzhou, Linz, Marrakech, Pekín, Seúl, Sapporo, Tianjin, Tokio Turín. Las entrevistas fueron realizadas, según la disponibilidad del entrevistado por teléfono o por correo electrónico, con preguntas en torno a funciones y estructura organizacional, presencia del destino en los medios sociales, uso interactivo y relacional y credibilidad e impacto de los medios sociales. Finalmente, accedieron a responder 9 destinos, lo que supone una tasa de respuesta del $56 \%$ sobre el objetivo inicial.

Los entrevistados fueron M. A. (Turín), G. F. (Pekín), R. D. (Buenos Aires), E. M. (Linz), J. H. (Málaga), T. E. S. (Copenhague), Z. J. (Hangzhou), S. V. M. (Amberes) e I. C. (Bogotá), que aparecen citados en este texto como E.1, E.2, E.3, E4, E.5, E.6, E.7, E8, y E.9.

\section{RESULTADOS}

La relevancia de la comunicación parece ya plenamente asumida por los responsables de los principales destinos urbanos del mundo. Al menos, todos los OMD tienen un departamento propio de Comunicación, que depende directamente de la dirección y participa del proceso estratégico de toma de decisiones. La estructura interna de estos departamentos puede variar de un destino a otro, aunque todos asumen las funciones de relaciones con la prensa, la comunicación corporativa, el diseño gráfico y el marketing (incluido el marketing digital). El tamaño del departamento suele estar vinculado al tamaño del destino, aunque en todos la plantilla está formada por una media de 10 personas. Destaca, sin embargo, el departamento de Comunicación del OMD de Copenhague, que está formado por un total de 25 personas. 
La importancia que los destinos conceden a la comunicación se refleja también en la formación de sus responsables. Todos los dircom son profesionales de la Comunicación, con licenciaturas, grados, diplomaturas en diferentes remas de este ámbito académico. También coinciden todos los destinos analizados en situar a sus dircom a las órdenes

directas de la dirección general, y entre sus funciones principales está el diseño, la coordinación y la preparación de las estrategias de comunicación, la articulación de pautas para el diseño de publicaciones, el liderazgo de los equipos y el asesoramiento a la dirección general en los temas relacionados con el ámbito comunicativo.

"La dirección es quien decide los temas principales que hay que comunicar, así como los contenidos digitales o los vídeos que deben usarse". (E.7)

"Validar, organizar y coordinar todas las campañas y elementos de comunicación, tanto online como offline. Esas son las funciones principales del director de comunicación en nuestra organización". (E. 8)

"Como director de comunicación, mis funciones son liderar y planear las estrategias de comunicación y divulgación de Bogotá como destino turístico; asesorar y articular las pautas de diseño de publicaciones, boletines y demás piezas de divulgación de actividades del Instituto; liderar el equipo de comunicaciones para generar información que pueda ser divulgada en el medio digital y asesorar al director general para un eficiente y productivo manejo del sistema interno en lo referente a internet, redes sociales, teleconferencias y televisión". (E.9)

Todos los directores de comunicación se encargan también de la gestión de los medios sociales, aunque llama la atención que los dircom de las oficinas de gestión turística de Amberes, Bogotá y Turín aseguren que no tienen responsabilidad sobre la gestión de las marcas de sus destinos. Igualmente, todos los destinos cuentan ya con planes estratégicos de comunicación y para establecer las estrategias y contenidos de los medios sociales tienen ejes temáticos específicos, reflejados o no, en manuales de estilo y de imagen corporativa. 
Pastor-Marín, Francisco-Manuel, Paniagua-Rojano, Francisco-Javier, y Cabrera-González, María-Ángeles

Todos los destinos cuentan, dentro de su departamento de Comunicación con un equipo específico dedicado a la gestión de los medios sociales, aunque algunos destinos (como es el caso de Málaga) lo tiene externalizado. Por lo general, estos equipos de medios sociales están formados por entre dos y seis personas. El perfil del gestor de medios sociales es variado (hay casos incluso en los que se admite la falta de formación), aunque mayoritariamente tienen estudios relacionados con la comunicación y el marketing, completados a posteriori con cursos de medios sociales, community manager, gestión web, etc.

\footnotetext{
"Usamos Facebook, Twitter, Instragram, Youtube y Linkedin porque son las redes sociales más usadas del mundo". (E.1)

"Usamos Facebook, Twitter, Instagram y Youtube porque son los principales medios para llegar a nuestro público potencial y poder así posicionar Buenos Aires como destino". (E.3)
}

"Realmente, la selección de medios sociales en los que estar presente se hizo mediante ensayo y error". (E. 8)

Facebook es, con diferencia, el medio social más utilizado. Todos los destinos tienen presencia en la mayor red social del mundo. Tras Facebook, se sitúan Twitter, Youtube, y, cada vez más, Instagram. A mayor distancia se encuentran Linkedin, Pinterest, Weibo, Webchat o Xing. Copenhague es el destino que está en un mayor número de medios sociales (7) y, por el contrario, Pekín sólo está en Facebook.

"Para la formulación de contenidos tenemos en cuenta siempre los temas y los públicos: al dirigirnos a diferentes públicos necesitamos optimizar lo más posible los contenidos que seleccionamos". (E.3)

"Normalmente, sobre los comentarios de los usuarios, los temas más recurrentes son comentarios positivos de orgullo de destino por parte de los visitantes y residentes o sorpresa y estímulo de los que no lo conocen". (E.5) 
La selección, según coinciden todos los entrevistados, se hizo teniendo en cuenta el número de usuarios de cada medio, aunque los responsables de comunicación de Bogotá y Buenos Aires apuntan también que eligieron los medios en los que están presentes por la capacidad que tienen para segmentar a los públicos.

Todos aseguran, además, que los diferentes medios sociales les ofrecen distintos resultados y la gran mayoría señala beneficios claros en su uso: fundamentalmente visibilidad, interacción, construcción de la imagen y promoción del destino, compromiso y generación de comunidad, aunque llama la atención que sólo uno de los destinos cite la mejora de la reputación de marca entre estas ventajas competitivas. Los responsables de comunicación de Amberes, Copenhague, Hangzhou y Málaga observan riesgos en el uso de los medios sociales debido, principalmente, al uso indebido de los datos o los comentarios críticos contra el destino, aunque ninguno de ellos se ha planteado dejar de usar un medio debido a estos riesgos.

Los directores de comunicación señalan que, con respecto a los medios sociales, sus funciones principales pasan por el control, la supervisión, la planificación, el análisis y la investigación. Los contenidos son decididos de forma mayoritaria por los propios gestores de medios o se extraen directamente de la web y sólo Copenhague y Linz asegura que son resultado de un trabajo en equipo. Eso sí, el $100 \%$ de los directores admite que hay unas estrategias definidas. Bogotá, por ejemplo, asegura que mensualmente presenta una estrategia de contenidos de acuerdo a los resultados registrados en el mes procedente.

"El mal uso de los datos es el principal problema que puede darse con respecto a los medios sociales".(E.4)

"Creo que los mayores riesgos de las redes sociales están en el mal uso de los datos y los comentarios críticos".(E.6)

Entre las principales directrices establecidas por las direcciones de los OMD para el uso de los medios sociales se citan la actualización continua de los contenidos, la protección de la marca del destino, el incentivar las noticias positivas, la interacción con los públicos 
Pastor-Marín, Francisco-Manuel, Paniagua-Rojano, Francisco-Javier, y Cabrera-González, María-Ángeles

y el no entrar en descalificaciones o insultos con aquellos usuarios que realicen comentarios negativos. De hecho, más de la mitad de los destinos analizados filtran los comentarios y todos, excepto Málaga, aseguran que eliminan aquellos que son considerados inapropiados (insultos, comentarios irrelevantes o spam). Los temas tratados en los comentarios se refieren, de forma mayoritaria, a los horarios de los monumentos y los detalles sobre algunos productos turísticos o muestran sorpresa ante aspectos del destino que desconocían.

En general, los dircom no saben cuantificar el tiempo dedicado a la gestión de los medios sociales, aunque la media es de 12 horas al día. Todos aseguran que se responde a los comentarios de los usuarios y la mayoría de ellos mantiene que lo hace antes de las 24 horas. Además, todos excepto Bogotá, Linz y Hangzhou tienen estrategias de gestión de la reputación en los medios sociales. Igualmente, todos excepto Hangzhou aseguran que pregunta a sus usuarios. Los públicos a los que se dirigen son turistas, viajeros potenciales, residentes en el destino, empresas comercializadoras o instituciones del sector turístico, por ese orden. No todos los entrevistados quisieron responder al presupuesto que destinan a la gestión de los medios sociales, aunque entre los que contestaron el intervalo oscila entre el $5 \%$ y el $60 \%$ del total del presupuesto del departamento.

"Nunca hay que descuidar la audiencia ni hay que subestimarla. Tenemos que ofrecer formatos nuevos y mantenerlos". (E.3)

"Comuníquese a la altura de los ojos, verifique cada publicación, no insulte a nadie. Sobre esas patas se asientan nuestras directrices". (E.4)

Sobre los motivos por los que están en los medios sociales, la mayoría de los dircom admite que es por difundir información sobre el destino o por difundir la marca, aunque cuando se les pregunta por estos contenidos en concreto la marca pasa a un tercer lugar, tras los atractivos turísticos del destino o la agenda cultural. También son motivos reseñados para estar presentes el hecho de estar a la vanguardia, conocer las opiniones de los viajeros, facilitar su participación o promover la fidelización. Casi la mitad añade también que están en los medios sociales "porque todos lo hacen". Además, destacan 
de forma mayoritaria los que aseguran sentir una presión externa para estar en estos medios y todos coindicen en que los ciudadanos lo demandan.

En cualquier caso, todos los responsables de comunicación de los destinos estudiados coinciden en la importancia de los medios sociales como instrumentos de comunicación y mantienen que sus organizaciones apuestan de forma decidida por el desarrollo digital como estrategia de penetración en el mercado. Además, coinciden en resaltar la apuesta por la tecnología vinculada a soluciones que aporten un valor añadido y mantienen que registrar la cantidad de información, analizarla y utilizarla de manera correcta será la clave para la supervivencia de los destinos turísticos. De hecho, los dircom mantienen que el big data tiene un valor fundamental para entender qué quiere el turista y poder ofrecérselo, por lo que se ha convertido en una herramienta clave para hacer rendir esa información y definir una oferta personalizada.

"Aprovechamos los comentarios para desarrollar nuestras estrategias y nuestros productos" (E.1)

"Analizamos los intereses, los tipos de reacciones y comentarios, tanto positivos como negativos y hasta los horarios en los que se realizan los comentarios" (E.3)

En este sentido, es un objetivo generalizado entre los directores de comunicación de los grandes destinos urbanos buscar una interacción con el cliente con el objetivo de dialogar; para ello-reconocen- los medios sociales son de gran utilidad, ya que permiten gestionar la imagen de la organización y del destino y proporcionan información de interés de los viajeros, sus gustos y necesidades, el grado de satisfacción o la fidelidad de estos turistas.

Pese a ello, los responsables de comunicación de estos destinos admiten que aprender a usar los medios sociales con estos objetivos no ha sido un proceso tan fácil como esperaban y siguen encontrando ciertas dificultades a la hora de obtener la información que necesitan. Tampoco está realmente claro el número de seguidores/fans que quieren conseguir en los diferentes medios e incluso la mitad de los entrevistados no 
Pastor-Marín, Francisco-Manuel, Paniagua-Rojano, Francisco-Javier, y Cabrera-González, María-Ángeles

terminan de ver si los esfuerzos realizados en medios sociales están siendo compensados.

Más de la mitad de los dircom ve en el bajo coste de los medios sociales un importante atractivo para apostar por esta forma de comunicación. En cualquier caso, todos reconocen que no es el económico el principal motivo para los medios sociales. No en vano, destacan que sus organizaciones tienen una cultura corporativa participativa, dan una gran importancia al uso de los medios sociales, no temen las interacciones con los usuarios y facilitan los recursos necesarios para tener una presencia activa en estos medios. Como resultado de ello, admiten que los medios sociales están contribuyendo a la fidelización de los viajeros.

"La comunicación a través de los medios sociales es la forma más económica y sencilla de llegar a cualquier potencial cliente en cualquier parte del mundo". (E.5)

\section{CONCLUSIONES}

El objetivo de este trabajo era conocer cómo los destinos gestionan los medios sociales, así como el perfil de sus responsables, sus funciones y la valoración de su presencia en la red. En ese sentido, podemos concluir que, a pesar de lo indicado por Huertas (2014), se ha avanzado mucho en la formación de los directores de comunicación, si bien aún queda camino por recorrer en cuanto a la formación de los gestores de los medios sociales. Quizá por ello la decisión sobre los contenidos sigue siendo muy jerárquica, siendo más responsabilidad de los directores que de los equipos que gestionan los medios sociales. Además, aunque todos los destinos tienen planes estratégicos, coincidimos con Hyas, Page y Buhalis (2013) en que el uso de los medios sociales es aún muy intuitivo. En línea con la propuesta de Paniagua y Huertas (2018), los destinos analizados empiezan a avanzar en la interacción y el diálogo, realizando preguntas que permitan una verdadera segmentación de los públicos.

En este sentido, los comentarios de los viajeros empiezan a ser tenidas en cuenta para generar nuevos contenidos, convirtiéndose así en herramientas de relaciones públicas, 
como ya avanzaban que debería hacerse Wigley y Lewis (2012) o Mariné-Roig y Huertas (2016). En cualquier caso, si bien sigue existiendo un cierto miedo a las críticas, como apuntaba Huertas (2014), los comentarios negativos ya han dejado de ser vistos como un problema sin solución y ningún destino se plantearía hoy dejar de estar en los medios sociales. De hecho, las ventajas son hoy mucho más reseñables que los riesgos, por lo que esta herramienta, como indicaban Zerfass et al. (2017), es ya el canal más importante para que los directores de comunicación de las Organizaciones de Marketing de los Destinos (OMDs) se dirijan a sus públicos. Además, los medios sociales empiezan a seleccionarse de forma cuidadosa. Sin embargo, aunque los medios sociales tienen una gran capacidad de contribuir al establecimiento de la marca del destino, como indicaban Domínguez y Araujo (2012), ésta no es aún uno de los principales elementos de comunicación. Es más, la comunicación de marca sigue siendo minoritaria y se mantiene como uso principal de los medios sociales informar sobre los atractivos turísticos.

Por ello, coincidimos con Paniagua y Huertas (2018) en la necesidad de que las OMDs ajusten más sus contenidos a las necesidades de los turistas. Es evidente que hay que establecer directrices más claras sobre la marca, aún minoritarias, y seguir trabajando en la comunicación emocional. Entendemos que este estudio aporta una nueva visión sobre el uso de los medios sociales en los destinos, al concluir que estamos en un momento de inflexión en el que se ha avanzado en la profesionalización de los responsables de estos medios y en el uso que hacen de ellos.

\section{REFERENCIAS BIBLIOGRÁFICAS}

Altamirano, V., y Túñez-López, M. (2016). Promoción y difusión turística en los países de Iberoamérica a través de los medios sociales. [Promotion and tourist diffusion in the countries of Ibero-America through social media]. International Journal of Information Systems and Tourism (IJIST), 1(1), 76-90.

Álvarez Díaz, Y., Pérez González, D. y Solana González, P. (2013). Contribución de la web 2.0 al desempeño organizacional en las empresas del sector turismo. 
Pastor-Marín, Francisco-Manuel, Paniagua-Rojano, Francisco-Javier, y Cabrera-González, María-Ángeles

[Contribution of web 2.0 to organizational performance in companies in the tourism sector]. Puente. Revista Científica, 7(2), 7-13.

Buhalis, D., y Law, R. (2008). Progress in information technology and tourism management: 20 years on and 10 years after the Internet - the state of eTourism research. Tourism Management, 29(4), 609-623.

De San Eugenio, J. (2011). Comunicación y Territorio. [Communication and Territory]. En De San Eugenio, J. (Ed.), Manual de comunicación turística: de la información a la persuasión, de la promoción a la emoción, (pp.53-72). Girona: Documenta.

Domínguez, T., y Araújo, N. (2012). El fenómeno 2.0 en el sector turístico. El caso de Madrid 2.0. [The phenomenon 2.0 in the tourism sector. The case of Madrid 2.0]. Pasos, 10(3), 225-237.

Fernández-Cavia, J. (2011). Ciudades, regiones y países como marcas: luces y sombras del place branding. [Cities, regions and countries as brands: lights and shades of place branding]. En J. De San Eugenio (Ed.), Manual de comunicación turística: de la información a la persuasión, de la promoción a la emoción (pp.103-113). Girona: Documenta

Fernández-Cavia, J., y Huertas, A. (2009). City brands and their communication through websites: identification of problems and proposals for improvement. En T. Torres y M. Gascó (Eds.), Information communication technologies and city marketing: Digital opportunities for cities around the world (pp.1174-1297). New York: IGI Global.

Fernández-Cavia, J., y Huertas, A. (2014). La gestión de las marcas de destino y de territorio desde la perspectiva de las relaciones públicas. [The management of destination and territory brands from the perspective of public relations]. Comunicació: Revista de Recerca i d'Análisi, 31(1), 9-26.

Hays, S., Page, S. J., y Buhalis, D. (2013). Social media as a destination marketing tool: its use by national tourism organisations. Current issues in tourism, 16(3), 211-239. 
Pastor-Marín, Francisco-Manuel, Paniagua-Rojano, Francisco-Javier, y Cabrera-González, María-Ángeles

Huertas, A. (2014). La comunicación de los territorios, los destinos y sus marcas. Guía práctica de aplicación desde las relaciones públicas. [The communication of territories, destinations and their brands. Practical guide of application from public relations]. Barcelona: DIRCOM-UOC.

Huertas, A., Setó, D., y Míguez, M. I. (2015). Comunicación de destinos turísticos a través de medios sociales. [Communication of tourist destinations through social media]. El Profesional de la Información, 24(1), 15-21.

Hvass, K. A., y Munar, A. M. (2012). The takeoff of social media in tourism. Journal of vacation marketing, 18(2), 93-103.

Lange-Faria, W., y Elliot, S. (2012). Understanding the role of social media in destination marketing. Tourismos: an international multidisciplinary journal of tourism, 7(1), 193-211.

Leung, D., Law, R., Van Hoof, H., y Buhalis, D. (2013). Social media in tourism and hospitality: A literature review. Journal of Travel \& Tourism Marketing, 30(1-2), 3-22.

Lim, Y., Chung, Y., y A Weaver, P. (2012). The impact of social media on destination branding: Consumer-generated videos versus destination marketer-generated videos. Journal of Vacation Marketing, 18(3), 197-206.

Lovejoy, K., y Saxton, G. D. (2012). Information, community and action: How non-profit organizations use social media. Journal of Computer-Mediated Communication, 17(3), 337-353.

Mangold, W. G., y Faulds, D. J. (2009). Social media: the new hybrid element of the promotion mix. Business Horizons, 52(4), 357-365.

Mariné-Roig, E., y Huertas, A. (2016). Metodologías de análisis. [Analysis methodologies]. En A. Huertas (Ed.), La comunicación de los destinos turísticos y sus marcas a través de los medios sociales (pp. 9-16). Tarragona: Universitat Rovira i Virgil. 
Pastor-Marín, Francisco-Manuel, Paniagua-Rojano, Francisco-Javier, y Cabrera-González, María-Ángeles

Martínez-sala, A. M., y Campillo-Alhama, C. (2018). La gestión de las redes sociales turísticas desde la perspectiva de las relaciones públicas 2.0: la importancia de diálogo. [The management of tourism social networks from the perspective of public relations 2.0: the importance of dialogue]. Revista Internacional de Relaciones Públicas, 8(16), 526.

Mendes Thomas, G., Augusto Biz, A., y Gândara, M. G. (2013). Innovación en la promoción turística en medios y redes sociales. Un estudio comparativo entre destinos turísticos. [Innovation in tourism promotion in media and social networks. A comparative study between tourist destinations]. Estudios y perspectivas en turismo, 22(1), 102-119.

Miguéns, J., Baggio, R., y Costa, C. (2008). Social media and tourism destinations: TripAdvisor case study. Advances in Tourism Research, 26(28), 1-6.

Míguez, M. I., Mariné, E., y Huertas, A. (2014). ¿Utilizan los destinos turísticos los medios sociales para crear diálogo con sus públicos? Estudio de los top post de Facebook y Twitter de los destinos turísticos españoles. [Do tourist destinations use social media to create dialogue with their audiences? Study of the top post of Facebook and Twitter of Spanish tourist destinations]. En TURITEC 2014. X Congreso de Turismo y Tecnologías de la Información y las Comunicaciones, (pp. 108-121). Málaga: Universidad de Málaga.

Mill, R. C., y Morrison, A. M. (2012). The tourism system. Dubuque, lowa: Kendall. Morgan, N., Pritchard, A., y Pride, R. (2011). Destination brands: Managing place reputation. Oxford: Butterworth-Heinemann.

Munar, A. M. y Jacobsen, S. J. K. (2014). Motivations for sharing tourism experiences through social media. Tourism Management, 43, 46-54.

Paniagua, F. J., y Huertas, A. (2018). El contenido de los medios sociales de los destinos turísticos y la búsqueda de información de los usuarios. [The content of social media of 
Pastor-Marín, Francisco-Manuel, Paniagua-Rojano, Francisco-Javier, y Cabrera-González, María-Ángeles

tourist destinations and the search for information of users]. Cuadernos de Turismo, $41,513-534$.

UNWTO \& WTCF (2017). City Tourism Performance Research. Madrid: UNWTO.

UNWTO (2018). UNWTO Annual Report 2017. Madrid: UNWTO.

Wichels, S. (2014). Cómo las redes sociales están cambiando la comunidad hotelera. [How social networks are changing the hotel community]. Revista Internacional de Tecnología, Ciencia y Sociedad, 3(2), 11-24.

Wigley, S., y Lewis, B. K. (2012). Rules of engagement: Practice what you tweet. Public Relations Review, 38(1), 165-167.

Xiang, Z., y Gretzel, U. (2010). Role of social media in online travel information search. Tourism Managament, 31(2), 179-188.

Xu, J. (2010). Social media influence on government affairs. Public Relations Magazine, $6(13)$.

Zerfass, A., Moreno, A., Tech, R., Vercic, D., y Verhoeven, P. (2017). European Communication Monitor 2017. Brussels: EACD / EUPRERA, Quadriga Media Berlin. 